\title{
Phenomenographic analysis and comparison of students' conceptual understanding of electric and magnetic fields and the principle of superposition
}

\author{
Esmeralda Campos@ \\ Tecnologico de Monterrey, Writing Lab, Institute for the Future of Education, \\ Av. E. Garza Sada 2501, Monterrey, 64849, Mexico \\ Eder Hernandez $\odot$ and Pablo Barniole \\ Tecnologico de Monterrey, Av. E. Garza Sada 2501, Monterrey, 64849, Mexico \\ Genaro Zavala๑* \\ Tecnologico de Monterrey, School of Engineering and Sciences, \\ E. Garza Sada 2501, Monterrey 64849, Mexico \\ Universidad Andres Bello, School of Engineering, Antonio Varas 880, Santiago 7500973, Chile
}

(Received 16 September 2020; accepted 21 June 2021; published 13 September 2021)

\begin{abstract}
There are studies of students' understanding of the concept of the electric field, the magnetic field, and the use of the superposition principle that have contributed to the creation of both educational strategies and assessment tools. However, the difficulties of these two concepts have not been compared comprehensively. Therefore, this study aims to compare students' conceptual understanding of electric and magnetic fields in questions regarding the field created by one source and the field produced by a system of two sources. We focus our study on students' explanations about the magnitude and direction of the field and their application of the superposition principle in both contexts (electric and magnetic). We conducted this study with 322 engineering students in a Mexican university. We designed two versions of an open-ended questionnaire, one with the context of electricity and the other with magnetism. We created the questions using the parallelism between electricity and magnetism and used schematic representations with similar surface features to represent this parallelism. Analyzing the data through a phenomenographic approach, the students' drawings and explanations gave insight into their understanding of the concept of field and the superposition principle application in the context of electricity and magnetism. We found that students have similar categories of understanding the concept of the electric and the magnetic field. In both contexts, there is an evident relationship between applying the principle of superposition and understanding the concept of field. We found that there is a greater tendency that a student correctly applies the superposition principle if that student has a robust representation of the field. We found evidence that students consistently merge two different representations (vector plots and field lines) in electricity and magnetism. We named this category of representation "hybrid vectors and field lines." We also found that some students who draw hybrid representations of the field can still apply the superposition principle correctly. However, some conceptual understanding difficulties are dependent on the context: the known difficulty of confusion between forces and fields is more attached to the electricity context. The confusion between electricity and magnetism concepts is more relevant in the magnetism context. As in other literature, we found more evidence that the correct answer in multiple-choice questions may lead students who have mild difficulties (such as memorization and creating a hybrid representation) to choose a correct answer. We recommend that teachers and researchers of electricity and magnetism acknowledge the relationship between the conceptual understanding of the field and applying the superposition principle. We suggest that instructors be attentive to how they approach using representations of electric and magnetic fields.
\end{abstract}

DOI: 10.1103/PhysRevPhysEducRes.17.020117

"genaro.zavala@tec.mx

Published by the American Physical Society under the terms of the Creative Commons Attribution 4.0 International license. Further distribution of this work must maintain attribution to the author(s) and the published article's title, journal citation, and DOI.

\section{INTRODUCTION}

Conceptual understanding of physics concepts and problem-solving skills at the university level are two of the general research topics in physics education research [1]. Research literature in conceptual understanding has 
long focused on identifying conceptual difficulties (i.e., misconceptions, alternative conceptions, and naïve conceptions). Many studies have identified students' conceptual difficulties in the context of undergraduate electricity and magnetism [2-4].

The literature on problem-solving skills has focused on the differences between novices and experts [1]. Studies have found that representational use has an important effect on developing problem-solving skills; novices tend to focus on the surface features of the representation of a physical situation, while experts focus on the underlying physical principles [5]. In the context of electricity and magnetism, the representation of the field may affect students' performance on magnetic force questions [6], and it may confuse the understanding of the field concept [7].

Even though electric and magnetic phenomena are undoubtedly different, they are ontologically related because the same sources (electric charge) under different conditions (static and dynamic) create them [8]. To an expert, the relation between these phenomena is evident in the parallelism of Maxwell's equations and the Lorentz force equation. These phenomena may also be related to a novice, but for their parallel surface features, instead of the underlying principles and meanings [5].

Epistemologically, there are different approaches in the teaching and learning of both phenomena and different difficulties for learning. When learning the electric field concept, students have difficulties adopting a way of thinking in terms of the field rather than interactions and confuse the concept of the electric field with electric force [9-12]. When learning the magnetic field theory, students have difficulties identifying the source of the magnetic field, and they often confuse the magnetic force and magnetic field [2]. Students have problems changing from the conception of interactions to the concept of field, which could be associated with an immature understanding of the electric and magnetic fields as vector fields [13].

The conceptual understanding of the field concept and the superposition principle has been studied in the electric and magnetic contexts. Still, the literature has not emphasized the comparison between these two contexts. There are several research-based assessments for students' understanding of general topics of electricity and magnetism, the Conceptual Survey of Electricity and Magnetism (CSEM) [3], the Brief Electricity and Magnetism Assessment (BEMA) [14], the Magnetism Conceptual Survey [15], and the Electricity and Magnetism Conceptual Assessment [16]. These assessments have evaluated, among other topics, the field concept and the application of the superposition principle, but they do not focus on a comparison between contexts.

These different studies that assess the conceptualization of the electric and magnetic field and the superposition principle application in both contexts give quantitative information about the difficulties that students may have when approaching these topics. More studies have explored the difficulties students have to apply the superposition principle, with qualitative $[12,17]$ and quantitative approaches [18-20]. Rainson, Tranströmer, and Viennot [18] found that some students can accept the existence of an electric field only if there is the possibility of motion. Other studies suggest that students think that only the nearest charges affect the electric field at the position in a situation of several charges and a position [12,19,20]. Furthermore, the characteristics of the representation may also affect the application of the superposition principle [17].

A possible source of difficulty for understanding the electric and magnetic field concepts is the confusion between them. Some students conceive magnetic fields as created by magnetic charges, similar to the electric field [21]. Some students claim that not-moving electric charges produce a magnetic field [2]. These difficulties suggest that students often confuse these topics that are ontologically related. However, only a few studies have approached the interference that might arise between the learning of electric and magnetic concepts $[4,22]$.

The comparison and analysis of interference between the electric and magnetic field concepts have been studied in terms of electric and magnetic forces when particles move through an electric or magnetic field $[4,23]$. Scaife and Heckler [4] found that students confuse the electric and magnetic phenomena of particles inside uniform electric and magnetic fields; this confusion is more prominent with the field line representation than with poles. The authors attribute students' confusion to the moments of evaluation, i.e., before or after the instruction of each relevant topic. We recently presented a comparison of students' difficulties on the notion of a charge (at rest and in motion) in an electric or a magnetic field [23]. Students' difficulties in the conceptual understanding of electric and magnetic phenomena have not been compared comprehensively for all the concepts related through parallelism. We are conducting several studies to contribute in this regard. In the present study, we focus on comparing students' understanding of the concepts of electric and magnetic fields and the superposition principle.

This study aims to compare students' conceptual understanding of electric and magnetic fields in questions regarding the field created by one source and the field produced by a system of two field sources through a phenomenographic lens. We focus our study on students' drawings and explanations about the magnitude and direction of the field and their application of the superposition principle in both contexts (electric and magnetic). We use a phenomenographic approach to analyze data because it provides deep insight into students' understanding while having many participants [24]. By analyzing open-ended questions, we can explore students' drawings and explanations without the guidance that multiple-choice questions may have on students' answers. The phenomenographic 
approach facilitates grouping students' answers into categories so that we can compare the two contexts. Our approach to make this comparison is novel because we draw upon the relation between electric and magnetic fields and the parallelism between their sources. We consider static point charges as electric field sources and dynamic point charges (electric currents) as magnetic field sources. How point charges and electric currents are often represented schematically is very similar, so we used these similarities to design our study; these schematic representations have been used in other studies about electric and magnetic concepts [3,14-16]. This comparison is relevant because it provides essential information about the difficulties in learning the concepts of electric and magnetic fields.

\section{METHODOLOGY}

We conducted a study with 322 engineering students in a large private Mexican university upon completing a calculus-based course on electricity and magnetism (E\&M). This course is the last of three introductory physics courses offered in this institution. The course consists of $3 \mathrm{~h}$ of lecture and $1.5 \mathrm{~h}$ of laboratory sessions per week. Students use a known textbook [25] and Tutorials for Introductory Physics [26]. The course is similar to an E\&M American university curriculum, in terms of content and duration [27].

We designed two versions of an open-ended questionnaire, one with the context of electricity and the other with magnetism. We created the questions using the parallelism between electricity and magnetism and used schematic representations with similar surface features to represent this parallelism. The instruments include four items: one question about the electric or magnetic field concept and one question about the superposition of the electric or magnetic field. We present a translation of the tests in Fig. 1 since we designed and administered them in Spanish. We administered the tests randomly to all of the students during

\section{Electricity}

1. The figure shows a point charge $+q$ and two positions in space represented by a cross (x). The two positions are at the same distance from the charge. Draw on the figure the electric field on the two positions. Explain your reasoning.

$\times$
$\times q \bullet$
ows two point charges $+q$ and $-q$.
re the electric field on the position

2. The figure shows two point charges $+q$ and $-q$. Draw on the figure the electric field on the position (x). Explain your reasoning.

2. The figure shows two long wires, one of them carries an outward $(\odot)$ and the other an inward $\left({ }^{\otimes}\right.$ ) electric current $+I_{0}$. Draw on the figure the magnetic field on the position (x). Explain your reasoning.

1. The figure shows a long wire carrying an outward $(\odot$ ) electric current $+I_{0}$ and two positions in space represented by a cross (x). The two positions are at the same distance from the wire. Draw on the figure the magnetic field on the two positions. Explain your reasoning.

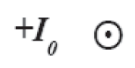

$\times$

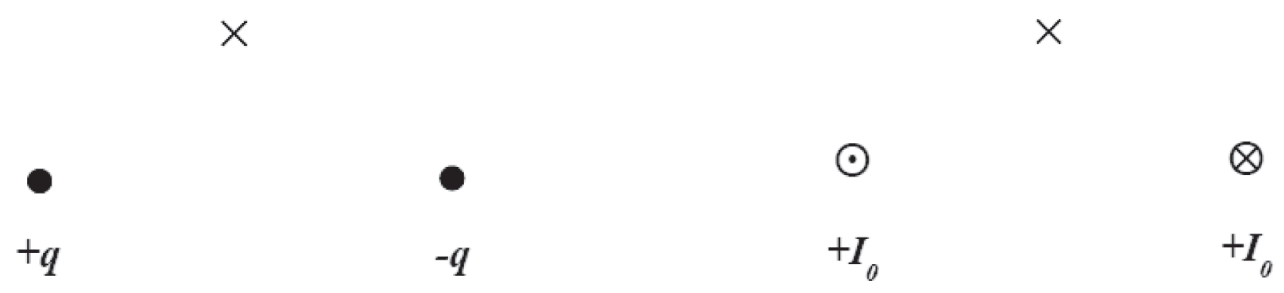

FIG. 1. The four questions analyzed in this study. On the left are the two items of the electricity version, and on the right, the same items of the magnetism version. The first question focuses on conceptual understanding of the electric and magnetics field's magnitude and direction, through their drawings and explanations. The second question focuses on applying the superposition principle in the context of electric and magnetic fields, respectively. 
(a)

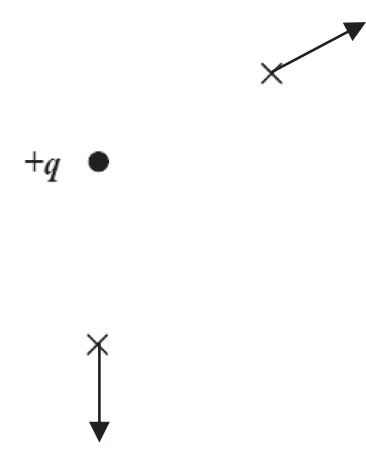

(c)

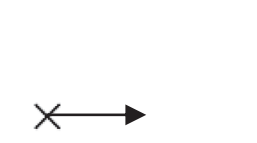

(b)

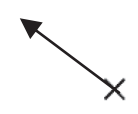

$+I_{0} \odot$

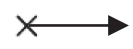

(d)

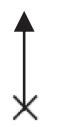

$+q$

$-q$

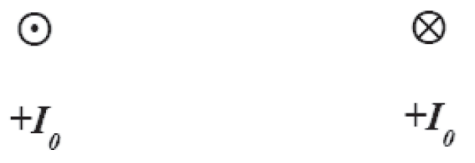

FIG. 2. The expected drawings for the questions of the field and the principle of superposition. On the left are those in the context of electricity. On the right, those in the context of magnetism. (a) The expected drawing for the electric field in question 1. (b) The expected drawing for the magnetic field in item 1. (c) The expected drawing for the electric field in question 2. (d) The expected drawing for the magnetic field in item 2 .

the last laboratory session of the semester, where 160 received the electricity version and 162 received the magnetism version.

As shown in Fig. 1, we decided to use similar representations for the sources: a small circle representing the point charge, the symbol for outward-pointing vectors representing the outward electric current, and the symbol for inward-pointing vectors to represent the inward electric current. These representations are customary in the electricity and magnetism classroom in textbooks $[26,28]$ and conceptual evaluations [3,14-16]. We represented the positions with crosses. We labeled the electric charge as $+q$ or $-q$, depending on the sign of the charge, and we marked the electric current as $+I_{0}$ to indicate that it is a conventional current. Our students are familiar with these representations by the end of the introductory electricity and magnetism course.

The first question is a system of only one source of the field, a point charge for the electric field, and a long straightline wire carrying an outward electric current for the case of the magnetic field. We asked the students to draw the electric field on the two positions marked in each situation. The positions are the same for both cases, one is directly below the field source, and the other is in a diagonal position, as shown. The problem explicitly stated that there is the same distance between the source of the field and the positions. The item asks students to explain their reasoning.
Since the nature of the electric and magnetic fields is different, we expect students to answer these two problems differently. The correct answer for the electric field is to draw electric field vectors that start at each position and point to the direction of a vector from the source to the position, $\hat{U}_{r-r^{\prime}}$. Since the problem states that the positions are the same distance from the point charge, the vectors should have the same length. We show this expected drawing in Fig. 2(a). The correct answer for the magnetic field is to draw magnetic field vectors that start at each position and point to the direction of $\hat{U}_{l \times\left(r-r^{\prime}\right)}$, with the same length for both vectors, see Fig. 2(b). The explanations may vary among students, which we analyze in the results section.

The second question is a system of two sources of field, a positive and a negative point charge for the electric field, and two long straight-line wires carrying an outward and an inward electric current, respectively, for the case of the magnetic field. We asked the students to draw the electric or magnetic field directly above the middle point between the two sources of the field. The position is the same for both situations. The question asked students to explain their reasoning.

In this part of the test, the two answers are different because of the nature of electric and magnetic fields. Still, the process to answer them should be the same: the application of the superposition principle. In the context 
of the electric field, the electric field vector points to the right. An example of this drawing is in Fig. 2(c). In the context of the magnetic field, the resulting vector points up, as shown in Fig. 2(d). The explanations may vary between students, but they account for how students apply the principle of superposition in the two contexts.

The questions presented in this study are reasonably fundamental. These questions do not provide a complete overview of student understanding of the field concept, which is very complex. With the analysis of students' answers to these questions, we aim to identify difficulties that may inhibit their learning of the field concept in the future. The introductory E\&M course provides a basic overview of the field concept in the electric and magnetic contexts. We think that if students can answer these basic questions, they have the opportunity to understand the field concept in more complex situations, such as applying the superposition principle [29]. However, if students present difficulties with such fundamental questions, they will probably drag these difficulties into their learning of the field concept in complex situations and more advanced specialized courses.

We analyzed the results with a phenomenographic approach. This approach allows us to understand how people experience and make sense of the physical world [24]. It recognizes that every person's experience and understanding are unique, but there are commonly shared experiences and conceptions. Therefore, it is possible to create categories that group individual conceptions into collective intellect [24]. This method has been used to analyze open-ended questions and derive students' understanding and difficulties when learning physical concepts [30,31].

We created the categories based on the answers from 20 students, reaching a consensus between experts. We applied the emerging categories to analyze the responses from all the students. When we identified the need to include new categories, we did so in an iterative process. We performed this analysis individually, by two of the authors, and compared our results. We used Cohen's kappa to measure the inter-rater reliability of our analysis, reaching an average of $95 \%$ agreement for the electricity version and $94 \%$ for the magnetism version.

\section{RESULTS}

We present our results focusing on the categories that emerged in the four questions. The categories provide an essential insight into the most distinctive characteristics that appear in students' answers. As Marton [32] explains, these categories are the most critical finding of phenomenographic research because, during the categorization process, we look for "structurally significant differences that clarify how people define some specific portion of the world" [32]; in this case, the concepts of the electric and the magnetic fields. Because the categorization process focuses on structural differences, some of the emerging categories may allow for comparisons between the understanding of the concepts of electric and magnetic fields.

To create our categories, we analyzed students' drawings and explanations. It is essential to analyze both drawings and explanations because students use representations to make meaning of the concepts under study. We first present the categories and results on the understanding of electric and magnetic fields in the first question and compare the two contexts. We later present the categories for applying the superposition of electric and magnetic fields in the second question and compare the two contexts. Finally, we present a crossed analysis for each version of the test, where we investigate how students' answers in the first and second questions relate. This analysis allows us to identify how the understanding and difficulties with the concept of field and applying the superposition principle may interact.

\section{A. Student understanding of the concept of field}

We identify the categories that emerged when analyzing students' understanding of the electric and magnetic field concept in question 1. It is essential to identify the drawings and the explanations that emerged separately. There are three categories of drawings of field that we could identify from students' answers. We present the drawings for the electric field in Fig. 3 and the magnetic field in Fig. 4.

In the drawing category "vector diagram," students draw a vector at each position with correct directions. The students representing the field with vector diagrams draw an arrow that starts at the positions marked with an $\mathrm{x}$. The arrow's length represents the magnitude of the electric or magnetic field at each position, which should be the same for both positions. The arrow's direction corresponds with the direction of the electric or magnetic field at each position. Figure 3(a) presents an example of this type of drawing in the electric field context, and Fig. 4(a) presents an example in the magnetic field context.

In the drawing category "hybrid vectors and field lines," students draw arrows that start at the charge and end at (or near) each of the two positions marked with an $\mathrm{x}$ in the electricity context. Figure 3(b) presents an example of this type of drawing. In most cases, students drew only the two arrows of interest. We consider this drawing as a hybrid between vectors and electric field lines because it shows the characteristic of vectors that it is only necessary to draw the vectors of interest while having the characteristic of electric field lines, that the arrows start at the source of the electric field. Only 5\% of students drew more arrows, resembling an incomplete electric field lines diagram. Students make variations of the vector diagrams and magnetic field lines in the magnetism question, often combining features of both representations. The variations are diverse, but many share some characteristics, such as curved arrows and open magnetic field lines [see Fig. 4(b)]. 


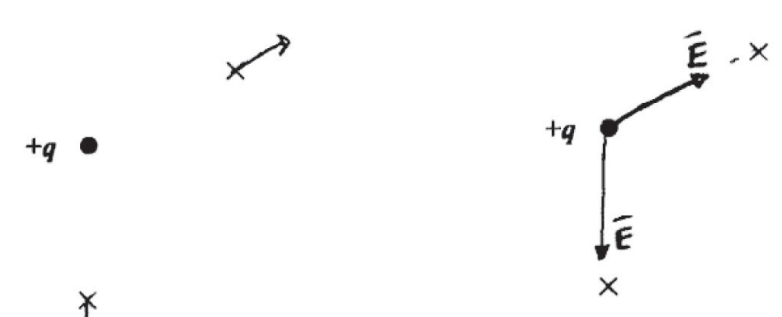

(a) (b)

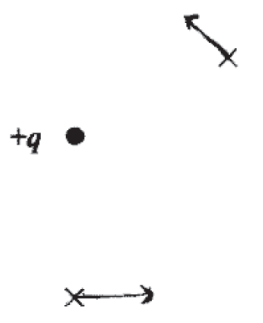

(c)

FIG. 3. We present examples of the drawings that students made in the first question of the electricity version. (a) An example of the drawing category vector diagram. (b) An example of the drawing category, hybrid vectors, and field lines. (c) An example of the drawing category, confusion between electric and magnetic fields.

In the drawing category "confusion between electric and magnetic fields," some students drew vectors or field lines that would correspond with the magnetic field generated by an outward electric current in the electricity context. Figure 3(c) presents an example of the vectors that correspond with the magnetic field in the electricity question. Some students drew vectors or field lines in the magnetism context that would correspond with the electric field generated by a positive charge [see Fig. 4(c)].

There are six different categories of the explanations given by students regarding their drawing of the electric field in the first question: "Field concept," "positive charges," "distance," "repulsion," "test charge," and "electric current and magnetic field." In the magnetism question, the six explanation categories are slightly different, which is natural because the contexts are different: "field concept," "right-hand rule," "positive, outward current," "distance," "relation to the electric field: attraction and repulsion" and "relation to the electric field: treats the wire as a positive point charge." there is an additional category, "other," which groups students whose explanations did not match any explanation categories and could not be classified. Students who did not answer the question were analyzed separately in an independent category, "does not answer." A detailed description of the categories of explanation is in the Appendix.

After identifying all drawing categories and the categories of explanation, we created the main categories by grouping drawings and explanations. The four categories that emerged were: vector field concept, hybrid vectors and field lines, confusion with electric interactions, and E\&M interference. We compare the categories for the understanding of electric and magnetic fields in Table I.

The categories vector field concept and hybrid vectors and field lines include variations of drawings and explanations that could imply a complete or partial understanding of the field created by one charge or current at this basic level. We noticed that the explanation field concept only emerged when students drew the vector diagram in both contexts. This result implies that the hybrid vectors and

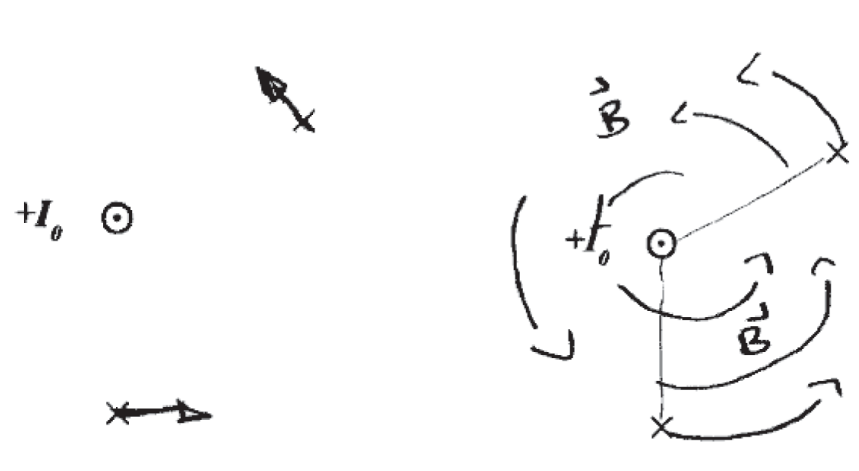

(a)

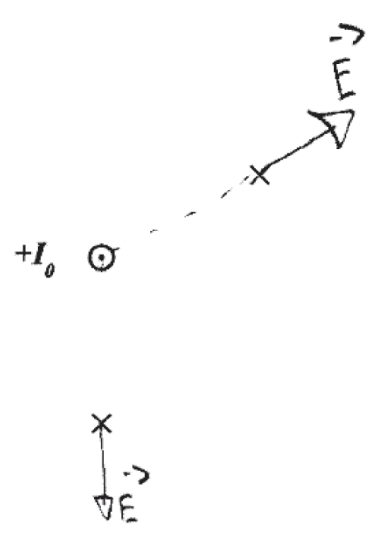

(c)

FIG. 4. Examples of the drawings that students made in the first question of the magnetism version. (a) An example of the drawing category vector diagram. (b) An example of the drawing category hybrid of vectors and field lines. (c) An example of the drawing category confusion between electric and magnetic fields. 
TABLE I. We compare the categories of understanding the field concept that emerged from students' drawings and explanations in the electric and magnetic contexts. In the magnetic context, the category confusion with electric interactions emerged combined with the E\&M interference; therefore, we included $4 \%$ of students in the latter.

\begin{tabular}{|c|c|c|c|}
\hline Category & Description & Electricity & Magnetism \\
\hline Vector field concept & $\begin{array}{l}\text { Complete understanding of vector field concept, } \\
\text { representation using the vector diagram }\end{array}$ & $41 \%$ & $48 \%$ \\
\hline Hybrid vectors and field lines & $\begin{array}{l}\text { Partial understanding of vector field concept, } \\
\text { variations of representation }\end{array}$ & $26 \%$ & $18 \%$ \\
\hline Confusion with electric interactions & Confusion between fields and interactions & $13 \%$ & $(4 \%)$ \\
\hline E\&M interference & Confusion between electric and magnetic fields & $6 \%$ & $14 \%$ \\
\hline Other & Other, unrelated, or does not answer & $14 \%$ & $20 \%$ \\
\hline Total & & $100 \%$ & $100 \%$ \\
\hline
\end{tabular}

field lines drawing might allow students to understand some of the characteristics of the field concept in the electric and magnetic context but does not stimulate relating the field concept with the corresponding physical principles. Students need to relate the field concept with physical principles to fully understand the field concept in complex situations in advanced courses.

The categories confusion with electric interactions and E\&M Interference imply difficulties in understanding the field concept at a basic level. The emergence of these difficulties from students' answers is coherent with the literature $[10,33]$. Furthermore, through our analysis, we were able to identify two different explanations that lead students to confuse the electric field and electric force. In the repulsion explanation, students explicitly state that there is repulsion between the point charge and the position, while in the test charge explanation, they confuse the position with a test charge. The category, E\&M interference, includes the students who confuse the electric field with magnetic field-related concepts, such as electric current and the right-hand rule. This difficulty is coherent with the findings reported by Scaife and Heckler [4] on the interference between the electricity and magnetism concepts after magnetism instruction.

From the results shown in Table I, we can observe that the category vector field concept is above $40 \%$ in both contexts. These results mean that more than $40 \%$ of students used a vector diagram to represent the electric or magnetic field while using an explanation coherent with the vector field concept. Similarly, the category hybrid vectors and field lines, combined with coherent explanations, is close to $20 \%$ in both contexts. The first category shows a complete understanding of the field created by one charge or current, while the latter offers a partial understanding. This result implies that there are difficulties related to the representation of vector diagrams and field lines, and the explanations might imply some memorization processes. Previous studies have reported the difficulties of combining characteristics of the vector diagram and the electric field lines diagram in the electric field context $[17,30]$. We observe that the difficulties of combining characteristics of the vector diagram and the electric field lines diagram seem to be independent of the context of electricity and magnetism.

The categories confusion with electric interactions and E\&M interference describe different difficulties. The confusion between the electric field and electric force has been studied before $[9,10,12,33]$. In this study, we identified that the category confusion with electric interactions is more prevalent in the context of electricity than in magnetism. This category emerged combined with the E\&M interference (4\% of students) in the magnetic context. There was a higher tendency to find students explaining that there would be attraction or repulsion in the electric field context. One possible explanation for this tendency is that in the electricity context, the electric field and the electric force are directly related through Lorentz's force, and the electric force is in the same direction as the electric field for positive test charges. Otherwise, in the magnetism context, the magnetic force is related to the magnetic field through a cross product, so the direction of the force is perpendicular to the direction of the field. Therefore, we only observed this confusion combined with the confusion between electric and magnetic fields. Some of the students who answered the question as if it were an electric field also answered that there would be repulsion or attraction.

Observe that the category $\mathrm{E} \& \mathrm{M}$ interference was more frequent in the magnetism context than in electricity. We did have a few students who answered the electric field question as if it were a magnetic field. Previous studies have observed that students answered electric force questions using the right-hand rule [4]. In this study, this difficulty emerged, but it was not as relevant as in the magnetism context. The difference could be that, in these questions, we asked students for the electric field instead of the electric force. In the magnetism context, the difficulty of answering the magnetic field question as an electric field was more prevalent. The percentage that we obtained (14\%) is similar to the results obtained by Scaife and Heckler [4].

\section{B. Application of the superposition principle in the context of electric and magnetic fields}

The categories in this section emerged from the analysis of students' drawings and explanations when answering 


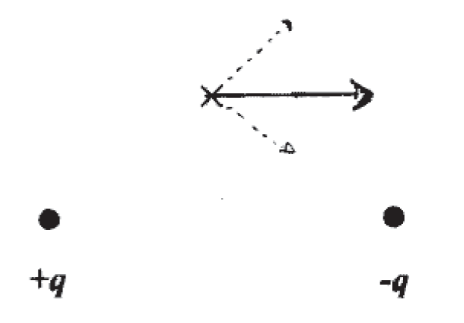

(a)

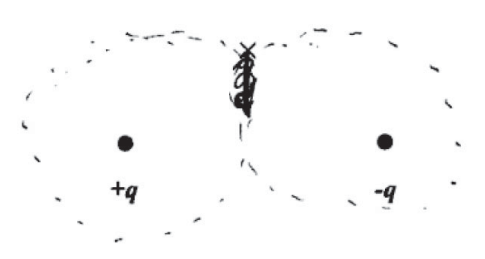

(d)

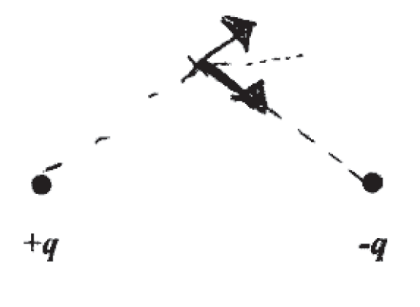

(b)

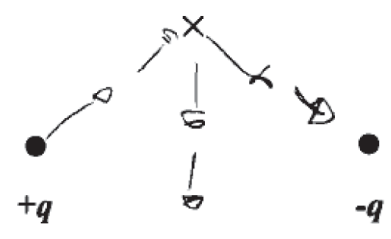

(e)

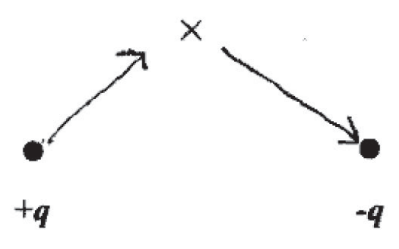

(c)

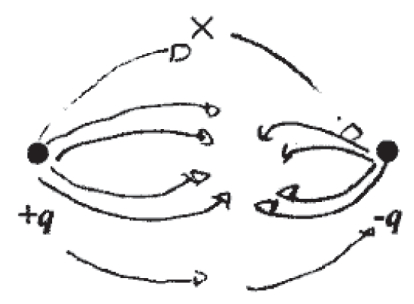

(f)

FIG. 5. Examples of the drawings that students made in the second question of the electricity version. (a) An example of the category superposition. (b) An example of the category contribution by each source. (c) An example of the category confusion force and field. (d) An example of the category E\&M interference. (e) An example of the category difficulties with graphic addition. (f) An example of the category other.

question 2 in the two contexts. The emerging categories describe how students apply the principle of superposition of the electric and magnetic fields. We present students' drawings of the superposition of the electric field in Fig. 5 and of the magnetic field in Fig. 6. By the end of the section, we compare the results from the two contexts.

In the category superposition, the students apply the superposition principle correctly. Some of them draw the vectors matching the contribution by each charge or wire and the resulting vector. Other students only draw the resultant vector. They explain that it is due to the superposition principle or by vector addition. For example, Fig. 5(a) shows a student who drew the vector corresponding to each of the charges with dotted lines and then drew the electric field vector pointing to the right. The student explained: "It is the resulting [vector] from the two charges that act on the position." Figure 6(a) shows a student's drawing in the magnetism context, the vector corresponding to the contribution by each of the wires with lighter arrows, and then the resulting magnetic field vector pointing upward. Students in this category explained that "Since they have the same magnitude, there is symmetry in the field so that only the $+y$ component remains." Even though these students do not cite the principle of superposition explicitly, their drawing and the explanation of a resulting vector are clues that imply the correct application of this principle.
In the category contribution by each source, the students identified through vectors or arrows the electric or magnetic field contributed by each charge or wire on the position $\mathrm{x}$. They did not add the two vectors graphically, nor did they mention the superposition principle. For example, in Fig. 5(b), a student draws the electric field vector generated by each charge on the position $\mathrm{x}$ in the electricity test. This student explained: "A positive charge has a negative electric field and the other positive." This explanation seems to refer to the contribution of the electric field by each of the charges; it relates to the direction as positive or negative, which is not an efficient way of describing directions. Figure 6(b) presents the drawing by a student who draws the magnetic field vector generated by each wire on the position $\mathrm{x}$. They explained that they used "the righthand rule." This explanation refers to the direction of the contribution of the magnetic field by each of the wires. In this category, students do not attempt to explain that there is a resultant vector of the electric field, nor do they mention the principle of superposition.

In the category confusion force and field, students explain through interactions between the charges or wires and the position, either attraction or repulsion. Some students do not aim to use the superposition principle to find the electric or magnetic field. For example, in Fig. 5(c), the student drew one arrow from the positive charge to the position marked by $\mathrm{x}$ and one arrow from the position to the 


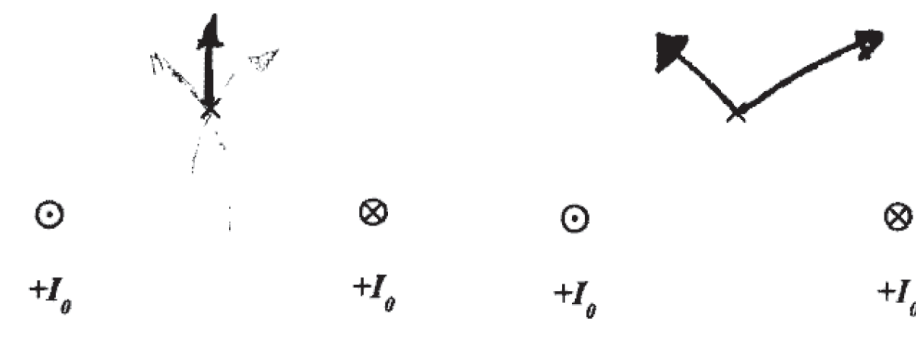

(a)

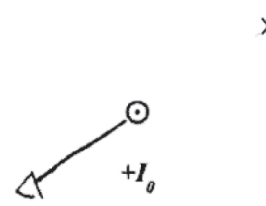

(d) (b)

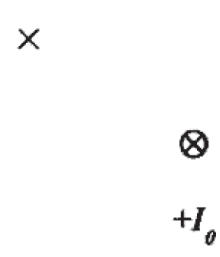

(e)

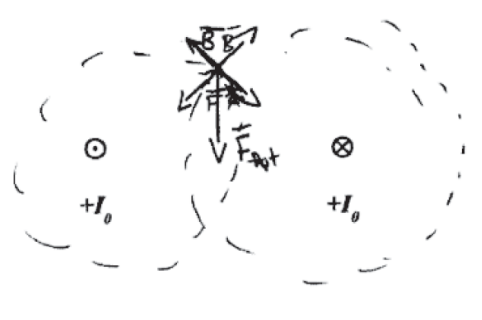

(c)

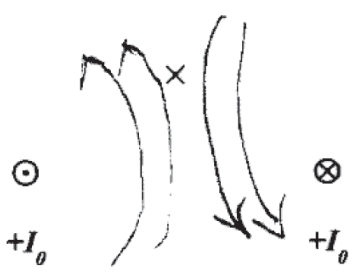

(f)

FIG. 6. Examples of the drawings that students made in the second question of the magnetism version. (a) An example of the category superposition. (b) An example of the category contribution by each source. (c) An example of the category confusion force and field. (d) An example of the category E\&M interference. (e) An example of the category vectors cancel. (f) An example of the category other.

negative charge. Students in this category explained that "one field repels the $x$ and the other attracts it." This response implies several difficulties in understanding the electric field; this student may confuse the position marked by $\mathrm{x}$ with an electric charge. In the magnetism test, a student presented the drawing in Fig. 6(c) and explained, "The magnetic force points downward since the sum of the magnetic forces of the two currents yields that."

In the category E\&M interference, students confuse electric charges with electric currents or electric fields with magnetic fields. For example, Fig. 5(d) shows the drawing by a student with two circles around the charges and a vector pointing downward in the electricity test. That student explained: "Is zero, both generate an electric field, but in opposite directions." From the drawing, the circles around the charges imply the confusion between electric and magnetic fields. For the magnetism test, Fig. 6(d) presents a student's drawing with two arrows that start at the current and point either to the $\mathrm{x}$ or away from it. The student explained that "The outward [current] has a direction opposite to the position because it is positive and outward, the inward [current] has a direction toward the position because it is positive and inward." From their drawing, the arrows that start at the wires with current are the features that imply the confusion between electric and magnetic fields.

In the category difficulties with graphic addition, the students apply the principle of superposition with difficulties. Some students draw arrows pointing from the charges to the position, which leads them to make the vector addition with some mistakes. Other students do not explicitly draw the contributions by each charge, so they make other mistakes that we cannot pinpoint with the available information. Figure 5(e) shows an example from a student. Figure 5(e) shows an example from a student who drew several vectors from each of the charges to the position. This student also drew vectors pointing downward and explained: "From one charge vectors leave and at the negative charge they enter so that the resulting vector will go down." Several students seemed to have the same difficulty when adding these vectors, which might be influenced by the bisector method for adding vectors graphically. This category did not emerge in the magnetic context.

In the category vectors cancel, the students explain that the magnetic field vectors cancel each other. For example, a student left their drawing blank, as shown in Figure 6(e), and explained that "There is no magnetic field, since the currents are equal, their magnetic fields are equal with opposite directions, so they cancel each other out." This response implies several difficulties understanding the magnetic field and the vector product between the current and the displacement vector. This category did not emerge in the electric context.

In the category other, the students drew variations of the electric or magnetic field or left their drawing blank. They provided explanations that do not answer the question or cannot be classified in any other categories. For example, a student drew a variation of the electric field lines of a 
TABLE II. We compare the different ways of applying the principle of superposition that emerged from students' drawings and explanations in the electric and magnetic contexts.

\begin{tabular}{|c|c|c|c|}
\hline Category & Description & Electricity & Magnetism \\
\hline Superposition & $\begin{array}{l}\text { The students apply the superposition principle correctly. } \\
\text { They explain that it is due to the superposition } \\
\text { principle or by vector addition. }\end{array}$ & $35 \%$ & $44 \%$ \\
\hline Contribution by each source & $\begin{array}{l}\text { The students identify the contribution of the field made by } \\
\text { each source but do not perform the addition or show a resulting vector. }\end{array}$ & $14 \%$ & $18 \%$ \\
\hline Confusion force and field & The students confuse the electric or magnetic field with its corresponding force. & $14 \%$ & $5 \%$ \\
\hline E\&M interference & The students confuse between electric and magnetic fields. & $5 \%$ & $6 \%$ \\
\hline $\begin{array}{l}\text { Difficulties with graphic } \\
\text { addition }\end{array}$ & $\begin{array}{l}\text { The students apply the superposition principle with difficulties. } \\
\text { The resulting vector is in a different direction. }\end{array}$ & $7 \%$ & $0 \%$ \\
\hline Vectors cancel & The students explain that the magnetic vectors cancel each other. & $0 \%$ & $9 \%$ \\
\hline Other & Other or unrelated & $22 \%$ & $13 \%$ \\
\hline No answer & Does not answer & $3 \%$ & $5 \%$ \\
\hline Total & & $100 \%$ & $100 \%$ \\
\hline
\end{tabular}

dipole, as shown in Fig. 5(f), and explained that "there is an electric field on $(x)$; however, the field is more focused between the two charges" in the electricity test. In the magnetism test, a student drew curved arrows near position $\mathrm{x}$ and explained: "the trajectory is from North to South," as shown in Fig. 6(f).

We present a comparison of the categories that emerged about the superposition principle application in the two contexts in Table II. We can observe that the context influences the difficulties in applying the superposition principle since the categories that emerged in both contexts have different percentages, and some categories emerged in only one of the contexts. The difficulties in understanding the electric and magnetic field concepts might influence the different categories.

In both contexts, the correct application of the superposition principle is higher than $35 \%$ of students. Some students attempt to apply this principle but do so incorrectly, having some difficulties with vector graphic addition (less than 10\%). As we can see, the main difficulty in applying the principle of superposition is the category contribution by each source, which is remarkable because these students identify that each source of electric or magnetic field contributes to the electric field at the position but does not proceed to carry out the vector sum. One reason could be that students perceive that identifying the contribution by each source is sufficient to indicate the field in the position.

\section{Analysis of understanding of the concept of field and the application of the superposition principle}

The analysis presents the relation between the first and second questions in each context. We measured the relationship between the two items by identifying the percentage of students who answered with a combination of the first and second questions, given the category of the first question [17]. In this analysis, we considered the percentages that resulted greater than $25 \%$ relevant for this study.

The category Vector field concept includes $41 \%$ of students in the electricity test and $48 \%$ of students in the magnetism version. We observe that $58 \%$ of the students with a vector field concept of the electric field apply the superposition principle effectively in the electricity test. In magnetism, $70 \%$ of the students with a vector field concept apply the superposition principle correctly. We highlight this tendency because it is remarkable that a complete understanding of the vector field concept promotes an efficient application of the superposition principle in both contexts. Similar behavior has been observed in the electricity context [17].

In the category hybrid vectors and field lines, there are $26 \%$ of students in the electricity version and $18 \%$ of students in the magnetism version. In electricity, we observed that $29 \%$ of students in this category apply the superposition principle, while $21 \%$ identified the contribution by each source without performing the vector addition. In magnetism, we can see that $26 \%$ of students with the hybrid of vectors and field lines category apply the superposition principle effectively, while $32 \%$ identified the contribution by each source. We can see that both contexts present a similar tendency so that students who draw hybrid representations of vector field plots and field lines may think of the contributions of the individual sources when answering a superposition question.

In the category confusion with interactions, we found $13 \%$ of students in the electricity context. We can see that students in this category tend to apply the superposition principle $(36 \%)$. However, $29 \%$ of students do not apply the superposition principle by explaining repulsion between the charges. We did not find significant relations in the magnetism version for this category.

In the category E\&M interference, there are $6 \%$ of students in the electricity version and $14 \%$ of students in the magnetism version. In electricity, we observe that $29 \%$ 
of students in the E\&M interference category in the first question remain in the E\&M interference category of the second question. In magnetism, we observe that $27 \%$ of students in this category in the first question correctly apply the superposition principle in the second question, while $36 \%$ remain with the $\mathrm{E} \& \mathrm{M}$ interference difficulty. We can see with this analysis that the difficulties of confusion between the electricity and magnetism contexts remain when applying the principle of superposition.

\section{DISCUSSION}

We present a discussion of the main results. First, we compare our results with items from known multiplechoice conceptual surveys in the contexts of electricity and magnetism with similar objectives. We then discuss the emergence of the hybrid representations, which combine features of vector plots and field lines, and how these representations have been reported in the literature. This hybrid representation emerged in both contexts, with a combination of different features. Finally, we highlight the main difficulties students have that might depend on context and contrast with the difficulties reported in the literature.

\section{A. Comparisons between the understanding of the field representation and the superposition principle in electricity and magnetism}

Throughout this contribution, we presented several comparisons between students' understanding of electric and magnetic vector field concepts, applying the superposition principle in both contexts, and the interaction between these two skills. It is interesting to see these comparisons because understanding the electric and magnetic fields and the superposition principle have been studied for each context independently in the physics education research literature. Being able to compare the way students make sense of these abstract physical quantities (i.e., the electric and magnetic fields), how they apply the principle of superposition in two contexts and how their understanding of the concept and the principle of superposition relate to each other gives us insight into a different way to approach the electricity and magnetism course.

For the electricity version, we did not find an item in the CSEM, nor the BEMA, that we could use to compare to our first item. The BEMA has two similar items (items 4 and 5), but they include an electric dipole, which requires a more complex type of answer; students need to apply the principle of superposition in combination with identifying the electric field. For this reason, in this section, we cannot present this comparison in the electric context. We provide the case of the magnetic context since the behavior of the two questions was similar. We think that the situation that we present in the first item of the electric field might be considered trivial; however, from our results, we believe that it is essential to identify the different ways in which students approach such a simple situation.

For the magnetism version, we found that item 26 of the CSEM is comparable to the first item of our study in the magnetic context. In our results, the first item of magnetism yielded three types of drawings, the correct vectors, the hybrid representation, and the confusion with the electric field. In the options of the CSEM, we can see that option (a) corresponds with the correct answer, which would be the vector drawing in our study. We can also identify that two options, (b) and (d), could be related to the confusion with the electric field since the vectors would point from the wire to each position or vice versa. Option (c) would most probably correspond with a mistake of direction while using the right-hand rule. It is interesting to see that three of the five options presented in the CSEM are consistent with our findings. However, due to the nature of open-ended questions, we were able to identify different types of drawings and a wide range of explanations for the possible correct answers.

In the CSEM, $67 \%$ of students chose the correct answer (post-test, calculus-based students) [3]. In comparison, in our study, we found $48 \%$ of students with the vector field concept and $18 \%$ of students with the hybrid representation (66\% of students between the two categories). We highlight the value of approaching these situations with open-ended questions since we found different types of representations that are not available in the multiple-choice instruments [3,14-16].

Students use representations to conceptualize the field concept [34]; when they are allowed to make their drawings to answer a question, some opt for a different representation (the hybrid between vectors and field lines). However, when they have options to adhere to, they tend to choose the option that more closely reflects their thinking. This premise implies that, in a multiple-choice test, when students select a correct answer, it does not always mean that students have the correct conception of the electric or magnetic field. In both contexts, we found that students tend to make hybrid drawings that blend features from the vector representation and the field line diagrams (we will further discuss this in the next section).

Furthermore, their explanations sometimes imply an incomplete description of the electric or magnetic fields. Some students describe only the direction when they explain that "the electric field is produced by a positive charge" and when they explain that "the magnetic field follows the right-hand rule." The explanations are essential in this type of question; it is not the same to appropriately use the right-hand rule (which is a mnemonic process) than to explain the relationship between the magnitude and direction of the field with the Biot-Savart law.

The superposition questions are comparable to item 9 in the CSEM for electricity, and item 23, for magnetism. In item 9, we observe that option (b) presents the application 
of the superposition principle with $62 \%$ of students, while options (c) and (d) present variations of confusion with interactions (26\% in total) [3]. In our results, we observe that $35 \%$ of students applied the principle of superposition, and $14 \%$ of students identified the contribution by each source (49\% in total). Only $14 \%$ of students presented the difficulty of confusing the superposition with electrical interactions.

In the magnetism context, we observe that in item 23 of the CSEM option (a) is the correct application of the superposition principle (63\%), options (b) and (c) most likely represent variations related to the confusion of electricity and magnetism (20\% in total), and choice (d) presents difficulties when applying superposition (8\%) [3]. In our study, we found that $44 \%$ of students applied superposition in this context, while $18 \%$ identified the contribution by each source (62\% of students between the two variations). Only $5 \%$ of students presented interference with electricity, and only $2 \%$ of students presented difficulties with graphic addition.

At least two steps are necessary to apply the superposition principle effectively: first, the student needs to identify the contribution that each source exerts on the electric or magnetic field; second, the student needs to add all the contributions in a vector sum. Our results suggest that, in an open-ended approach, some students make only the first step; they may deem the second step unnecessary. However, in multiple-choice tests, the options they have are of only one vector; they only consider the complete application of the principle (after the second step) and related difficulties. In the multiple-choice questionnaires that we analyzed, none of the options considers that students may identify the contributions by each source and not perform the addition [3,14-16]. When students see that the possible options only include one vector at the position, they conclude that they need to add the contributions by each source. Therefore, the possibilities of multiple-choice tests might help students to realize that they need to make the vector addition, which leads to more students choosing the correct answer.

\section{B. The hybrid representation of the electric or the magnetic field}

The drawings students make for the electric and magnetic field concepts provide insight into their understanding. The drawings that we found were very revealing because, in both contexts, we saw the typical vector diagram [Fig. 3(a) for the electric field and Fig. 4(a) for the magnetic field], but we also found that students make their diagrams that combine characteristics of the vector field plot and the field lines diagram, as shown in Figs. 3(b) and 4(b) for each context.

In electricity, they drew arrows that start at the positive charge and are directed towards the position where they mean to obtain the electric field. We had observed this type of diagram in a previous study [17]. We interpret this drawing as a hybrid between the electric field lines representation and the vector representation because it shares a few characteristics. In this drawing, the arrows start at the positive charge and end near the position or extend farther away, which is one of the rules to draw electric field lines. On the other hand, there are not enough arrows to represent the magnitude of the field with the density of field lines, so we think that in this case, the arrow's length denotes the electric field, which is a characteristic of the vector representation. The direction of the field would be the same in both representations in this particular case. Because of these blended features, we consider this drawing as a hybrid between the vector plot and the electric field lines diagram.

In magnetism, they drew several curved arrows or other variations that combine characteristics of the vector representation and the magnetic field lines. To the best of our knowledge, this hybrid representation of the magnetic field has not been reported in the literature; other studies have found that students interpret the field direction as the curved direction of field lines in the context of the electric field [12]. A similar representation has been reported by Ref. [35] of mathematical representations without physical context. We interpret this drawing as a hybrid between the magnetic field lines' representation and the vector representation because it shares a few characteristics different from those in the context of electric fields. In this drawing, the arrows start at the positions, as vectors would. The student does not seem to be very aware of the changes of magnitude because there is no pattern in the size of the arrow. The arrow's direction is curved, following the circles that magnetic field lines would trace. Because of these blended features, we consider this drawing and other similar ones as hybrids between the vector field plot and the magnetic field lines diagram.

We think that students who make these hybrid drawings in either context take some characteristics of each of the representations, probably those they find helpful or that they have memorized, and combine them to make meaning of the concept. This conception is what we refer to as the hybrid representation. Another aspect of this hybrid representation is that students often combine it with explanations that could also result from a rote learning process. Examples of these explanations are the use of the righthand rule in magnetism or that positive charges create radially outward electric fields in the context of electricity. Our results did not find any students who would make a hybrid drawing and explain the field concept using physical principles.

As mentioned earlier, multiple-choice tests do not include this drawing in their options [3,14-16]. One advantage of using open-ended questionnaires is the possibility to make these findings. It is crucial to identify the difficulties of understanding that may arise from using 
hybrid representations because they can be addressed explicitly through instruction. Since multiple-choice tests do not present this type of representation, it is not easy to detect these difficulties. When students do not see the option that they are thinking within the possible choices, they select the option that looks more similar to their thinking. At first glance, we may think that these students have a complete understanding of the field concept. However, through open-ended questionnaires and detailed analysis, we have confirmed that some students blend the features of different representations and make partial or rote learning explanations in both electric and magnetic contexts.

\section{Main conceptual difficulties found to be dependent on context}

Our results suggest the confusion between fields and forces is more persistent in the context of the electric field (13\% of our students presented this difficulty). Even in the context of the magnetic field, this difficulty implied a confusion between the magnetic field and electric force. So we see that electric interactions dominate this difficulty. This difficulty is well known in the literature in the electrical context $[9,10,12,33]$. Few studies have addressed this difficulty in the magnetic context [2]. A recent study [23] analyzed the interactions between positive charges in uniform electric and magnetic fields. In the electric field, we found a category for "impetus," which was not present in the context of the magnetic field. This result suggested that students carry the alternative conception of the preGalilean force and difficulties in applying the principle of superposition [23]. In this present study, we add to the discussion that the difficulties regarding the concept of force remain more strongly in the context of electricity and that the confusion with interactions affects the application of the superposition principle.

On the other hand, in the context of the magnetic field, the confusion between electricity and magnetism concepts is more relevant (14\% of our students presented this difficulty). This result was a surprising finding because the literature has suggested otherwise [4,23]. However, in previous studies, the physical situations had been interactions between charges and fields. Students are often more familiar with the interactions between charges in motion in uniform magnetic fields than in electric fields. In this study, we asked students about the electric field or the magnetic field created by a source, not about the interaction between a charge and an existing uniform field. We think this confusion was more prevalent in magnetism because students have already memorized how a positive charge creates the electric field. Therefore, only a small proportion of students (6\%) confused the electric charge with an electric current, and a larger proportion of students (14\%) confused the electric current with an electric charge.

\section{CONCLUSION}

The objective of this study was to compare students' conceptual understanding of the concept of electric and magnetic fields in questions regarding the field created by one source and the field created by a system of two sources. We analyzed students' answers to a question on the understanding of the concept of field and a question on applying the superposition principle in the contexts of electricity and magnetism independently. We used a phenomenographic approach to analyze students' drawings and explanations and create a hierarchical structure to compare contexts.

We found that students have similar categories of understanding the concept of the electric and the magnetic field. A complete understanding of the concept of field using the vector field concept category and the Hybrid vectors and field lines category do not have qualitative differences between contexts. However, the Vector field concept category is more frequent in both scenarios.

We compared our results with known conceptual surveys from the literature. We found that the proportion of correct answers in multiple-choice questions coincides with (i) the proportion of students in both the vector field concept and the hybrid vectors and field lines categories in the first question, and (ii) with the proportion of students who effectively apply the principle of superposition and the students who identify the contribution by each source in the second question. The correct answer in multiple-choice questions may lead students who have mild difficulties (such as memorization and creating a hybrid representation) to choose a correct answer; this makes these mild difficulties harder to detect. The effect of multiple-choice questions on students' correct answers has been studied in the context of astronomy; more students were able to choose the correct answer in a multiple-choice test than those who were able to explain their answer effectively [36]. It is also possible that the correct answer helps students to realize the missing steps that they need to apply in a superposition question. To confirm these hypotheses, we would need to conduct studies designed explicitly as multiple-choice tests that include the different representations that emerged with this open-ended approach and include incomplete applications of the superposition principle.

The emergence of the hybrid vectors and field lines category is a finding by itself. The particularity of merging two different representations (vector plots and field lines) in two different contexts (electricity and magnetism), resulting in representations that would not be correct in the eyes of an expert, but that allows students to make some sense of the physical situation. The explanations that emerge with the hybrid vectors and field lines category could result from memorization processes, not necessarily a fully developed field concept, which only emerged with the vector representation. 
The difficulties that emerged regarding the field concept are context dependent. Students have a higher tendency to answer with a confusion with electric interactions in the context of the electric field. In contrast, in the context of the magnetic field, students have a higher tendency to present $\mathrm{E} \& \mathrm{M}$ interference. This result is relevant since it might indicate that we prefer making our instruction focused on interactions in the electricity curriculum and less on the magnetic counterpart. It could be understandable since the introduction of the electric field is done through Coulomb's law (force) and that for the magnetic field, it is usually done through Bio-Savart's law (not force).

We found that the differences in applying the superposition principle do not depend on the context of electricity and magnetism. In both contexts, there is an evident relationship between applying the principle of superposition and the understanding of the concept of field. We found that the more significant proportion of students in the Vector field concept results in the correct application of the principle of superposition. We also found that students who draw hybrid representations can apply the superposition principle correctly. Still, there is a tendency to identify the contribution made by each charge without making the vector sum. Additionally, we found that the confusion between fields and forces is consistent with applying the principle of superposition but is more prominent in the electricity context. We finally observed that the difficulty of confusing electricity and magnetism is persistent in both contexts.

The results from this investigation provide essential information regarding the understanding that students have of the concepts of electric and magnetic fields and the application of the principle of superposition. Some implications for the teaching and learning of these concepts that derive from this study is that there should be more emphasis in the vector representation to create a fully developed field concept that considers the relation between the definition of the field and the Lorentz force, and the Coulomb's and Biot-Savart's laws. Instructors should also consider that some typical phrases that we regularly use in class get attached to students' language, even though they might not necessarily interiorize the concepts, which results in the repetition of rules that do not imply understanding. In this effort, instructors should also clearly establish and highlight the differences between forces and fields and between the phenomena of electricity and magnetism.
Our study has several limitations. When interpreting students' answers to open-ended questions, there might be a gap between what students choose to share in their drawings and explanations and how they conceive each phenomenon. Therefore, the analysis we performed is limited by the evidence that we get only from students' drawings and explanations. It is also essential to recognize that the hierarchical structure of the categories is formed through interpretation done by the researchers and is therefore limited by the access that we have to students' answers and our own experiences. The phenomenographic approach used in this study allowed us to find variations of drawings and explanations that would not be available with a multiple-choice approach. However, having open-ended questions also allows more room for dispersing answers that could be regarded as noise.

From these limitations, there is an opportunity for further research. It would be interesting to complement this kind of comparison with multiple-choice questionnaires since the results are not prone to the researchers' interpretation; they allow statistical analyses and reduce noise. If the aim is to deepen into students' thinking, it would also be interesting to interview them regarding electric and magnetic fields, the principle of superposition, interactions, and other fundamental concepts and principles in the electricity and magnetism curriculum. There is yet much to explore around the comparisons between the understanding of electricity and magnetism concepts. The parallelism between concepts proposed in this study is one of the possible approaches to this kind of comparison. This kind of comparison could also be transferred into different contexts of physics where parallelism between concepts exists.

\section{ACKNOWLEDGMENTS}

We acknowledge the financial and technical support of Writing Lab, TecLabs, Tecnologico de Monterrey, Mexico, in the production of this work.

\section{APPENDIX}

We present the categories of explanation that emerged in the first question of electricity in Table III and of magnetism in Table IV.

TABLE III. We present the categories of explanation in the first question of the electric context.

\begin{tabular}{llc}
\hline \hline Category & \multicolumn{1}{c}{ Description } & Example \\
\hline Field concept & $\begin{array}{c}\text { The students state that a test charge would exhibit } \\
\text { a force (of repulsion) if it were placed at the positions } \\
\text { marked. This is considered the electric field concept } \\
\text { because it relates to the Lorentz equation, } \mathrm{E}=\mathrm{F} / \mathrm{q}\end{array}$ & $\begin{array}{c}\text { "If there were a positive test charge, it would } \\
\text { move in the direction of the force because } \\
\end{array}$ \\
\hline
\end{tabular}


TABLE III. (Continued)

\begin{tabular}{|c|c|c|}
\hline Category & Description & Example \\
\hline Positive charges & $\begin{array}{l}\text { The students in this category of explanation stated } \\
\text { that positive charges have an outward electric field }\end{array}$ & $\begin{array}{l}\text { "The electric field vectors will be pointing } \\
\text { outward since the charge is positive } \\
\text { and with the same magnitude." }\end{array}$ \\
\hline Distance & $\begin{array}{l}\text { The students in this category explained that the } \\
\text { electric field magnitude is the same at both } \\
\text { positions because they are at the same distance } \\
\text { from the charge }\end{array}$ & $\begin{array}{l}\text { "The electric field at both positions has } \\
\text { the same magnitude because they are at the same } \\
\text { distance, just in a different direction." }\end{array}$ \\
\hline Repulsion & $\begin{array}{l}\text { The students in this category of explanation } \\
\text { stated that there is repulsion between the } \\
\text { positive charge and the positions }\end{array}$ & $\begin{array}{l}\text { "The arrows are of the same magnitude } \\
\text { and with direction opposite to } \\
\text { the charge because }+q \text { repels the two positions." }\end{array}$ \\
\hline Test charge & $\begin{array}{l}\text { The students in this category of explanation } \\
\text { treated the position as a test charge, which implied } \\
\text { that there would be repulsion }\end{array}$ & $\begin{array}{l}\text { "I considered the } x \text { as a positive sign }+, \\
\text { the electric field at the two positions } \\
\text { would point outward." }\end{array}$ \\
\hline $\begin{array}{l}\text { Electric current } \\
\text { and magnetic } \\
\text { field }\end{array}$ & $\begin{array}{l}\text { The students in this category made drawings } \\
\text { and explanations that correspond with the drawing } \\
\text { category "Confusion between electric } \\
\text { and magnetic fields," which implies } \\
\text { confusion with the magnetic } \\
\text { field created by an outward electric current }\end{array}$ & "Right-hand rule." \\
\hline
\end{tabular}

TABLE IV. We present the categories of explanation in the first question of the magnetic context.

\begin{tabular}{|c|c|c|}
\hline Category & Description & Example \\
\hline Field concept & $\begin{array}{l}\text { The students in this category of explanation } \\
\text { stated that the magnetic field is the same } \\
\text { at both positions because they are at the } \\
\text { same distance, and the direction is given } \\
\text { by a cross product and the right-hand rule. } \\
\text { In their explanation, the student emphasized } \\
\text { the current and the vector between the position } \\
\text { and the current. The combination of these } \\
\text { explanations adds to a complete understanding } \\
\text { of the Biot-Savart law. }\end{array}$ & $\begin{array}{l}\text { "I used the right-hand rule for a wire } \\
\text { transporting current to get the direction of the } \\
\text { field, which is counter-clockwise. I got the } \\
\text { direction from there. There should also be a } \\
90^{\circ} \text { angle between the line from the wire to the } \\
\text { point and the direction of the field. They have } \\
\text { the same magnitude because they are at the } \\
\text { same distance." }\end{array}$ \\
\hline Right-hand rule & $\begin{array}{l}\text { The students in this category of explanation } \\
\text { stated that they used the right-hand rule to } \\
\text { get the direction of the magnetic field at } \\
\text { both positions. They do not refer to the } \\
\text { magnitude of the magnetic field. }\end{array}$ & $\begin{array}{l}\text { "The wire has an outward current; using } \\
\text { the right-hand rule, you can know } \\
\text { the direction of the magnetic field." }\end{array}$ \\
\hline $\begin{array}{l}\text { The positive, } \\
\text { outward current }\end{array}$ & $\begin{array}{l}\text { The students in this category of explanation } \\
\text { stated that positive outward currents have } \\
\text { a counter-clockwise magnetic field }\end{array}$ & $\begin{array}{l}\text { "Since the current on the wire is outward, } \\
\text { the magnetic field is counter-clockwise; } \\
\text { therefore, it has a given direction } \\
\text { in the positions." }\end{array}$ \\
\hline Distance & $\begin{array}{l}\text { Both positions are at the same distance from } \\
\text { the wire. The students in this category } \\
\text { explained that the magnetic field magnitude } \\
\text { is the same at both positions because they } \\
\text { are at the same distance from the wire. }\end{array}$ & $\begin{array}{l}\text { "Using the right-hand rule and knowing } \\
\text { that the two positions are at the } \\
\text { same distance, we know that the magnetic } \\
\text { field is the same in both points } \\
\text { and is counter-clockwise." }\end{array}$ \\
\hline $\begin{array}{l}\text { Relation to the electric } \\
\text { field: Attraction and } \\
\text { repulsion }\end{array}$ & $\begin{array}{l}\text { The students in this category of explanation } \\
\text { stated that there is repulsion between } \\
\text { the positive outward current and the positions }\end{array}$ & $\begin{array}{l}\text { "The current, being }+ \text { [positive], } \\
\text { repels the point charges }(x) "\end{array}$ \\
\hline $\begin{array}{l}\text { Relation to the electric } \\
\text { field: Treats the } \\
\text { wire as a positive point } \\
\text { charge }\end{array}$ & $\begin{array}{l}\text { The students in this category of } \\
\text { explanation stated that positive } \\
\text { charges create an outward magnetic field. }\end{array}$ & $\begin{array}{l}\text { "The magnetic field in a positive charge of } \\
\text { this type causes a magnetic field } \\
\text { outward from the cable." }\end{array}$ \\
\hline
\end{tabular}


[1] J. L. Docktor and J. P. Mestre, Synthesis of disciplinebased education research in physics, Phys. Rev. ST Phys. Educ. Res. 10, 020119 (2014).

[2] J. Guisasola, J. M. Almudí, and J. L. Zubimendi, Difficulties in learning the introductory magnetic field theory in the first years of university, Sci. Educ. 88, 443 (2004).

[3] D. P. Maloney, T. L. O'Kuma, C. J. Hieggelke, and A. V an Heuvelen, Surveying students' conceptual knowledge of electricity and magnetism, Am. J. Phys. 69, S12 (2001).

[4] T. M. Scaife and A. F. Heckler, Interference between electric and magnetic concepts in introductory physics, Phys. Rev. ST Phys. Educ. Res. 7, 010104 (2011).

[5] M. T. H. Chi, R. Glaser, and E. Rees, Expertise in problem solving, Advances in the Psychology of Human Intelligence, edited by R.J. Sternberg (Lawrence Erlbaum Associates, Hillsdale, NJ, 1982), Vol. 1.

[6] T. M. Scaife and A. F. Heckler, The effect of field representation on student responses to magnetic force questions, AIP Conf. Proc. 951, 180 (2007).

[7] S. Törnkvist, K.-A. Pettersson, and G. Tranströmer, Confusion by representation: On student's comprehension of the electric field concept, Am. J. Phys. 61, 335 (1993).

[8] D. J. Griffiths, Introduction to Electrodynamics, 4th ed. (Cambridge University Press, Cambridge, England, 2018).

[9] X. Bohigas and M. C. Periago, Alternative mental models of second-year engineering students for coulomb's law and the electric field, Rev. Electron. Investig. Educ. 12, 1 (2010), http://redie.uabc.mx/vol12no1/contenido-bohigas .html.

[10] C. Furió and J. Guisasola, Difficulties in learning the concept of electric field, Sci. Educ. 82, 511 (1998).

[11] A. Garza and G. Zavala, Contrasting students' understanding of electric field and electric force, AIP Conf. Proc. 1513, 142 (2013).

[12] J. Li and C. Singh, Investigating and improving introductory physics students' understanding of the electric field and superposition principle, Eur. J. Phys. 38, 055702 (2017).

[13] M. Saarelainen, A. Laaksonen, and P. E. Hirvonen, Students' initial knowledge of electric and magnetic fieldsmore profound explanations and reasoning models for undesired conceptions, Eur. J. Phys. 28, 51 (2007).

[14] L. Ding, R. Chabay, B. Sherwood, and R. Beichner, Evaluating an electricity and magnetism assessment tool: Brief electricity and magnetism assessment, Phys. Rev. ST Phys. Educ. Res. 2, 010105 (2006).

[15] J. Li and C. Singh, Developing and validating a conceptual survey to assess introductory physics students' understanding of magnetism, Eur. J. Phys. 38, 025702 (2017).

[16] M. W. Mccolgan, R. A. Finn, D. L. Broder, and G. E. Hassel, Assessing students' conceptual knowledge of electricity and magnetism, Phys. Rev. Phys. Educ. Res., 13, 020121 (2017).

[17] E. Campos, G. Zavala, K. Zuza, and J. Guisasola, Electric field lines: The implications of students' interpretation on their understanding on the concept of electric field and the superposition principle, Am. J. Phys. 87, 660 (2019).
[18] S. Rainson, G. Tranströmer, and L. Viennot, Students' understanding of superposition of electric fields, Am. J. Phys. 62, 1026 (1994).

[19] L. Viennot and S. Rainson, Design and evaluation of a research-based teaching sequence: the superposition of electric field, Int. J. Sci. Educ. 21, 1 (1999).

[20] C. Singh, Student understanding of symmetry and Gauss's law of electricity, Am. J. Phys. 74, 923 (2006).

[21] D. P. Maloney, Charged poles?, Phys. Educ. 20, 310 (1985).

[22] M. H. P. Kesonen, M. A. Asikainen, and P. E. Hirvonen, University students' conceptions of the electric and magnetic fields and their interrelationships, Eur. J. Phys. 32, 521 (2011).

[23] E. Hernandez, E. Campos, P. Barniol, and G. Zavala, The effect of similar surface features on students' understanding of the interaction of charges with electric and magnetic fields, Proceedings of the Physics Education Research Conference 2019, Provo, UT, 2019 (2020), https://www .compadre.org/per/items/detail.cfm?ID=15279.

[24] F. Marton, Phenomenography-Describing conceptions of the world around us, Instr. Sci. 10, 177 (1981).

[25] H. D. Young and R. A. Freedman, University Physics with Modern Physics, 13th ed. (Pearson, Mexico, 2013).

[26] L. C. McDermott and P. S. Shaffer, Tutorials in Introductory Physics (Pearson Education, Buenos Aires, 2001).

[27] P. Barniol, E. Campos, and G. Zavala, Conceptual Survey of Electricity and Magnetism: Analysis of the items and recommendations for improvement, Proceedings of the Physics Education Research Conference 2016, Sacramento, CA, 2016 (2016), p. 44, https://www.compadre .org/per/items/detail.cfm?ID=14189.

[28] R. Chabay and B. Sherwood, Matter and Interactions (John Wiley \& Sons, New York, 2015).

[29] E. Campos and G. Zavala, A look into students' interpretation of electric field lines, in Handbook of Research on Driving STEM Learning With Educational Technologies, edited by M. Ramírez-Montoya (IGI Global, Hershey, PA, 2017), https://doi.org/10.4018/978-1-5225-2026-9 .ch017.

[30] E. Campos, G. Zavala, K. Zuza, and J. Guisasola, Students' understanding of the concept of the electric field through conversions of multiple representations, Phys. Rev. Phys. Educ. Res. 16, 010135 (2020).

[31] K. Zuza, P. Van Kampen, M. De Cock, T. Kelly, and J. Guisasola, Introductory university physics students' understanding of some key characteristics of classical theory of the electromagnetic field, Phys. Rev. Phys. Educ. Res. 14, 020117 (2018).

[32] F. Marton, Phenomenography-A research approach to investigating different understandings of reality, J. Thought 21, 28 (1986), http://idr.uin-antasari.ac.id/ 473/1/kualitatifrisetEDUCATION.pdf\#page $=151$.

[33] A. Garza and G. Zavala, Electric field concept: Effect of the context and the type of questions, AIP Conf. Proc. 1289, 145 (2010).

[34] E. Campos, G. Zavala, K. Zuza, and J. Guisasola, Students' conversion from electric field line diagrams to other representations, Proceedings of the Physics Education Research Conference 2019, Provo, UT, edited by Y. Cao, S. Wolf, and M. B. Bennett (AIP, New York, 
2019), https://www.compadre.org/per/items/detail.cfm?ID= 15259 .

[35] L. Bollen, P. van Kampen, C. Baily, M. Kelly, and M. De Cock, Student difficulties regarding symbolic and graphical representations of vector fields, Phys. Rev. Phys. Educ. Res. 13, 020109 (2017).
[36] M. M. Wooten, A. M. Cool, E. E. Prather, and K. D. Tanner, Comparison of performance on multiple-choice questions and open-ended questions in an introductory astronomy laboratory, Phys. Rev. ST Phys. Educ. Res. 10, 020103 (2014). 